\title{
REGENERACIÓN DE EMPRESAS DE LA ECONOMÍA SOCIAL: UN RETO EN EL PAÍS VASCO
}

\author{
POR \\ Baleren BAKAIKOA AZURMENDI ${ }^{1}$, \\ Enekoitz ETXEZARRETA ETXARRI ${ }^{2}$ y \\ Jon MORANDEIRA ARCA ${ }^{3}$
}

\section{RESUMEN}

Las organizaciones en general a lo largo de su existencia experimentan un proceso de degeneración de los principios por los cuales fueron creadas. Lo mismo les ocurre a las organizaciones pertenecientes a la Economía Social. A este respecto existe abundante literatura explicando este fenómeno degenerativo de las empresas de la Economía Social. Sin embargo, en el País Vasco se están dando varios casos en los que se busca regenerar dichas empresas, mayoritariamente cooperativas, tratando de implementar y reestablecer los principios y valores por las que fueron creadas. Además son empresas de la Economía Social de envergadura como son el Grupo Eroski, S. Cooperativa, Irizar, S. Cooperativa y el Grupo GBE-NER. Estos tres casos serán analizados en el presente trabajo.

Palabras clave: degeneración y regeneración de principios cooperativos, organizaciones de la Economía Social, grupos.

\section{Claves Econlit: P130}

\footnotetext{
${ }^{1}$ Baleren BAKAIKOA AZURMENDI: Director de GEZKI. Universidad del País Vasco (UPV/EHU). Dirección de correo electrónico: baleren.bakaikoa@ehu.es

${ }^{2}$ Enekoitz ETXEZARRETA ETXARRI: Investigador de GEZKI. Universidad del País Vasco (UPV/EHU). Dirección de correo electrónico: enekoitz.etxezarreta@ehu.es

${ }^{3}$ Jon MORANDEIRA ARCA: Investigador de GEZKI. Universidad del País Vasco (UPV/EHU). Dirección de correo electrónico: jon.morandeira@ehu.es
}

REVESCO No 112 - Extraordinario en Homenaje al Profesor Alfonso Carlos MORALES

GUTIÉRREZ - ISSN: 1885-8031 - www.ucm.es/info/revesco

http://dx.doi.org/10.5209/rev_REVE.2013.v112.43064

Fecha de recepción: 26/12/2012

Fecha de aceptación: 04/04/2013 


\title{
REGENERATION OF THE SOCIAL ECONOMY ENTERPRISES
}

\begin{abstract}
The organizations in general throughout their existence suffer a process of degeneration of the principles by which they were created. The same phenomenon happens to the organizations belonging to the Social Economy. In this respect, there also exists abundant Literature dealing with cooperatives. Nevertheless, in the Basque Country there are several cases in which they try to regenerate these companies, mainly cooperatives, trying to implement and to re-establish the principles and values by which they were created. Among these companies, some of them are of great relevance like Eroski Group, Irizar, S. Cooperativa and GBE-NER Group. These three groups will be analyzed in this research work.
\end{abstract}

Key words: degeneration and regeneration of cooperative principles, Social Economy organizations, groups.

\section{INTRODUCCIÓN}

Es sabido que las organizaciones nacen, se desarrollan, se estabilizan y con el transcurrir del tiempo se degeneran. Esta es la historia de las organizaciones. La Iglesia de Cristo tuvo un inicio democrático y después un desarrollo esplendoroso y luego vino su declive ¿definitivo? Es posible, aunque en esta institución se dé un nuevo proceso renovador y pueda adaptarse a las nuevas circunstancias, sin embargo difícilmente llegará a ser una escuela de la democracia, al estilo de lo que observó Alexis de Tocqueville en su viaje a Norteamérica. Así mismo los partidos socialdemócratas, comunistas, etc. también tuvieron sus años de democracia, gloria e influencia, hasta que su afán a ocupar puestos de influencia, dichos partidos se han convertido en apéndice de los partidos neoliberales, siendo difícil distinguir sus políticas económicas de las aplicadas por la derecha neoliberal. Lo mismo podemos decir de los sindicatos obreros más poderosos.

Las primeras instituciones de la Economía Social como las empresas cooperativas nacieron a mediados del siglo XIX, respondiendo a las duras condiciones impuestas por el sistema capitalista. Los pioneros de Rochdale crearon la primera cooperativa en bases a unos REVESCO No 112 - Extraordinario en Homenaje al Profesor Alfonso Carlos MORALES GUTIÉRREZ - ISSN: 1885-8031 - www.ucm.es/info/revesco 
principios cooperativos que han servido como guía para las cooperativas que han surgido en todo el planeta. Estos principios fueron adoptados por la Alianza Cooperativa Internacional (ACI) en 1895 en su reunión fundacional. Cien años más tarde, en su congreso centenario celebrado en Manchester (RU) en 1995, respondiendo a la evolución del movimiento cooperativo, el eslogan utilizado tenía el sugerente título Making Membership Meaningful. De esta forma, se pretendía realizar ciertos ajustes en el camino recorrido por las cooperativas en las que sus miembros cada día pierden protagonismo, reduciendo su perdiendo interés por el proyecto empresarial, en beneficio de la tecnoestructura.

Este proceso degenerativo de las cooperativas ha sido expuesto por Victor Pestoff (2009) en Suecia donde en 1991, las cooperativas de consumo se estructuraron en base a ordinarias relaciones comerciales, en lugar de relaciones democráticas de intercooperación y solidarias, tal como contemplan los principios de la ACI. Más tarde, en 2001, en la era de la globalización, las cooperativas de consumo de Dinamarca, Noruega y Finlandia se unieron con el fin de proteger su mercado escandinavo de la competencia exterior, pero diluyendo el espíritu cooperativo.

Los kibbutz israelíes cuando se fundaron fueron democráticos, solidarios y autogestionarios. Sin embargo, con el tiempo han perdido el principio de solidaridad y se asemejan a empresas capitalistas (Singer, 2008). La democracia real de una empresa requiere una participación activa en la toma de decisiones y no es suficiente votar en la Asamblea General de la cooperativa una vez al año, sino que se debe participar en todas las decisiones que afectan a la empresa (Defourny y Thomas, 1986). Esta participación se debe hacer extensiva a los stockholders para que la democracia sea más completa (Ugarte, 2004).

También la literatura marxista establece que a largo plazo las cooperativas se degeneran como organizaciones democráticas como consecuencia de presiones exógenas y otras veces por fuerzas endógenas. En este sentido Meister $(1974,1984)$ argumenta que a largo plazo las organizaciones democráticas llegan a ser controladas por una élite dirigente o, utilizando la terminología de Galbraith (1967), por la tecnoestructura. Este parece ser el destino de las organizaciones en general y también de las empresas de la economía social incluidas las cooperativas. 
La tesis de la degeneración de las cooperativas, teorizada por parte autores internacionales (DiMaggio y Powell, 1983; Evers y Laville, 2004) fue ratificada también para el caso vasco (Errasti et al, 2003 y Bakaikoa et al, 2004), aunque había posibilidades para su regeneración, para la cual se proponían varias fórmulas. Más tarde (Bakaikoa, et al, 2008) consideraron que uno de los pilares del desarrollo económico del País Vasco era la profundización en la participación de los socios de trabajo y de sus empleados por cuenta ajena. En este sentido, se demostraba que había varias cooperativas de trabajo asociado que habían cambiado de rumbo, servían de ejemplo de organizaciones que aumentando el nivel de participación, elevando su democratización, se convertían en empresas más eficientes.

Siguiendo a Cornforth (1988) la degeneración de las cooperativas se da por tres razones. En primer lugar se da la degeneración formal o constitucional cuando las cooperativas se transforman en sociedades capitalistas en las que los trabajadores pierden algunos de sus derechos laborales. En segundo lugar cuando las cooperativas sustituyen sus fines iniciales por maximizar los beneficios. Y en tercer lugar, la degeneración de la organización se produce cuando la cooperativa pasa a estar controlada por un grupo reducido de dirigentes quienes constituyen la élite dirigente (Michels, 1949) o que Galbraith (1967) denominaría tecnoestructura.

Conrforth y Spear (2004) mantienen que los miembros de las cooperativas y mutuas y de los consejos rectores elegidos por dichos miembros pierden capacidad de incidir en la toma de decisiones de sus organizaciones empresariales a medida que éstas se hacen más grande. Esta pérdida de poder de los miembros de las cooperativas y mutuas y de sus consejos rectores es más acentuada que en las sociedades por acciones porque en éstas los accionistas velan más por sus intereses que en las empresas de la Economía Social donde el capital está subordinado al trabajo. Con el fin de superar la pérdida de influencia de los consejos rectores se propone aumentar las participación de las mujeres y jóvenes en los órganos de decisión, incluso hacerlo extensivo a otros stakeholders (Borzaga y Santuari, 2001; Borzaga y Depedri, 2012; Bakaikoa, 2008); en este sentido Spear (2004) y Spear, Cornforth y Aiken (2009) proponen en publicaciones posteriores medidas para controlar el poder de los gestores de las cooperativas y éstos actúen en sintonía con los miembros de estas organizaciones empresariales.

REVESCO No 112 - Extraordinario en Homenaje al Profesor Alfonso Carlos MORALES GUTIÉRREZ - ISSN: 1885-8031 - www.ucm.es/info/revesco 
Esta preocupación por recuperar el espíritu inicial del movimiento cooperativa la ha asumido el Consejo Superior de Cooperativas de Euskadi (CSCE) en su Plan Estratégica de 2009/2012 recuerda que este órgano público “asumirá, defenderá, promoverá asimismo los valores y principios cooperativos" (CSCE, 2009). Para ello, el Consejo se constituye en órgano de debate de estos principios y valores y establecerá medidas concretas para que las cooperativas vascas interioricen dichos principios y valores (CSCE, 2010). A través de la Confederación de Cooperativas de Euskadi y las respectivas federaciones se quiere llegar a todas las cooperativas vascas para que los socios actuales y futuros conozcan de primera mano la doctrina que conforma al movimiento cooperativo del País Vasco y la materialicen en la práctica diaria, frenado el deterioro de las reglas de funcionamiento de las cooperativas, especialmente de las grandes. El congreso de la Asociación de Sociedades Laborales de Euskadi (ASLE) celebrado en marzo de 2012 trató de reafirmar los principios de la Economía Social ante la posible deriva hacia planteamientos procapitalistas de algunos de sus afiliados.

La corporación Mondragón tampoco elude este problema de degeneración de las cooperativas. Este problema se agudiza en las filiales ubicadas en el extranjero, donde en algunos países no existe una legislación cooperativa y en otros se carece del mínimo espíritu cooperativo entre los trabajadores autóctonos contratados. Para implementar las ideas del cooperativismo, la corporación cuenta con el instituto Lanki de la Mondragón Unibertsitatea el cual trabaja en un proyecto de recuperación de los principios y valores cooperativos a nivel de los órganos de la corporación y de las cooperativas del grupo (Altuna, 2008; Azkarraga, 2007).

Algunas cooperativas de Mondragón tratan de contrarrestar la tendencia a la degeneración de las mismas. Para ello han reformulado las competencias de los consejos rectores y se ha implementado la autogestión, superando la participación en la propiedad y resultados, extendiéndose a la gestión donde la iniciativa la llevan los socios trabajadores. Este cambio de tendencia se está dando en Irizar, S. Coop. y en Ampo, S. Coop., cooperativas de trabajo asociado, desligadas de la corporación Mondragón en 2008. También, la cooperativa de distribución Eroski de este grupo trata de reconducir el nivel de participación de sus empleados, abriéndoles las puertas para acceder a ser socios trabajadores. Todo esto supone también la regeneración de los principios de la Economía Social, transformando los trabajadores por cuenta ajena es socios trabajadores. 
Otro aspecto a tener en cuenta en las empresas de la Economía Social, incluyendo las cooperativas, es que el capital está supeditado al trabajo. Las sociedades capitalistas reducen la plantilla en aras a obtener mayores beneficios, mientras que las cooperativas mantienen los puestos de trabajo, aunque para ello tengan que reducir los anticipos laborales (equivalente a salarios). Esta política laboral es habitual en las empresas de la Economía Social tal como está quedando patente en la recesión económica actual (2008-2013).

El objetivo de este artículo no es otro que dar a conocer algunas experiencias, no todas ellas cooperativas, de regeneración de los principios y valores asumidos por las entidades de Economía Social, especialmente respecto a la democracia y participación en la toma de decisiones.

El método empleado para realizar esta investigación ha consistido en recurrir a documentos como los Planes Estratégicos de 2006-2008 y 2009-2012 del Consejo Superior de Cooperativas de Euskadi, el congreso de ASLE de 2012, las publicaciones de la corporación Mondragón (TU-Lankide), trabajos realizados por investigadores de instituciones académicas del grupo y entrevistas con miembros de dicha corporación (Agirre, A., 2006, Ugarte, L. 2004). También el material publicado y recogido en la prensa escrita y en las páginas web de las organizaciones analizadas en el presente trabajo.

Asimismo, se han utilizado datos de los artículos publicados en revistas científicas por los autores de este trabajo de investigación. La información de los casos analizados corresponden al año 2006 para Irizar, 2010 para GBE-NER y 2011 para Eroski; a pesar de esta falta de sincronía, consideramos que el objetivo de esta investigación es coherente. Por otra parte, el artículo se ha estructurado con el estudio de tres casos, los cuales agrupamos en dos subgrupos: en primer lugar se analiza el grupo Eroski, perteneciente a la corporación Mondragón, donde a los trabajadores se les posibilita ser socios trabajadores, y en segundo lugar Irizar, S. Cooperativa y el grupo GBE-NER donde los trabajadores, socios o con contrato de trabajo por cuenta ajena, participan en la toma de decisiones, impulsando la democracia interna de la empresa. A continuación se exponen las conclusiones más destacadas de la investigación.

REVESCO No 112 - Extraordinario en Homenaje al Profesor Alfonso Carlos MORALES GUTIÉRREZ - ISSN: 1885-8031 - www.ucm.es/info/revesco 


\section{ESTUDIO DE CASOS DE REGENERACIÓN}

\subsection{EROSKI: cooperativización del grupo de distribución de Mondragón}

\section{a) Datos de interés}

La cooperativa Eroski fue fundada en 1969 consecuencia de la fusión de cinco cooperativas de consumo locales. Eroski está constituida por socios trabajadores y socios de consumo. Para 1972 esta cooperativa tenía 32 centros de venta y superaban los 17 mil socios de consumo. En 1980 alcanzaba los 100 mil socios de consumo, aproximadamente el 15\% del total de las familias del País Vasco. Cuatro años más tarde, en 1984, Eroski alcanza la cifra de 1.246 socios después de crear 168 nuevos puestos de trabajo. En 1987 incorpora a su rede cinco nuevos centros, iniciando la divulgación de la revista Eroski; a esta red propia hay que añadir los 176 establecimientos de Erosle. Los años noventa se destacan por el elevado crecimiento de hipermercados y otros centros, así como del empleo, incluyendo los años de depresión económica como fueron 1992 y 1993 (Bakaikoa, 1995).

La primera década del siglo XXI continúa por la senda de crecimiento y en 2008 cuenta con 115 hipermercados en territorio del estado español, 524 centros de dimensión media y 173 centros más pequeños, además de agencias de viaje, gasolineras, etc., sumando un total de 2.377 centros de negocio. Asimismo, inicia su expansión en Francia y Andorra, con 39 y 4 centros, respectivamente.

El empleo creado por Eroski durante la primera década del presente siglo sigue creciendo en términos similares al de la década anterior. Los puestos de trabajo se dividen entre los ocupados por los socios trabajadores y los contratados por cuenta ajena. Así, en 2008 de un total de 52.711 empleos, 14.733 correspondían a socios trabajadores y 37.978 a nosocios o contratados por cuenta de Eroski, sólo el 28\% eran socios trabajadores y el $72 \%$ contratados. Los socios de consumo superaban los 600 mil miembros.

El año siguiente, en 2009, consecuencia de la crisis y por la necesidad de hacer frente a los compromisos de pago por los préstamos recibidos por 21 entidades bancarias, el grupo Eroski ha eliminado 5.200 empleos eventuales y negociado con sus acreedores bancarios para retrasar su deuda hasta 2014. La reducción de la plantilla ha sido compensada con un aumento 
de la jornada laboral en un 10\%, sin variar los ingresos de los empleados. En 2011, la dirección ha propuesto someter a votación democrática a los socios aumentar un 5\% la jornada laboral a cambio de mantener los anticipos laborales (sueldos), lo que supone trabajar algo más de media hora más por jornada, o reducir en ese porcentaje dichos anticipos laborales. De los 14.500 socios cooperativistas, de un total de 47.500 empleados, el $90 \%$ optó por trabajar más tiempo y el 10\%, unos 1.500 cooperativistas, prefirieron ganar menos dinero; de esta forma, estos últimos pueden conciliar mejor su vida personal con la laboral. Sin duda, esta una prueba de la superioridad del cooperativismo en particular y de la Economía Social, en general, para mantener el nivel de empleo (Cinco Días, 19 y 20 de marzo).

\section{b) Regeneración del Grupo Eroski}

Ante la dualidad de la plantilla del Grupo Eroski, la dirección quiere ofrecer la oportunidad de ser socios trabajadores en condiciones económicas, sociales y políticas semejantes a los socios trabajadores tradicionales de Eroski, S. Cooperativa. Se trata de que todo el personal llegue a ser protagonista, propietario y participe en el proyecto empresarial. De esta forma, el futuro del grupo sería más viable que manteniendo el modelo actual. Asimismo, funcionario el criterio democrático de una persona un voto, independientemente del capital aportado por los socios.

El nuevo modelo del Grupo Eroski tiene como eje central la cooperativa matriz Eroski, S. Cooperativa, de la cual forman parte los 14.733 socios trabajadores. Esta cooperativa matriz formará parte de de las cooperativas mixtas, las cuales serán unidades de negocio independiente, con socios trabajadores y Eroski S. Cooperativa con el 50\% del capital social. No se creará una única cooperativa.

El grupo está regulado por el Estatuto Marco de la Estructura Societaria (EMES). Este estatuto es el texto legal que define la estructura societaria cuyo vértice es la cooperativa de segundo grado. EMES conduce a todas las cooperativas del Grupo Eroski.

Por su parte, el Grupo Eroski está formado por el denominado Ámbito Cooperativo el cual estructura todas las empresas cooperativas y las sociedades mercantiles donde la cooperativa matriz tiene participación en el capital. El Ámbito Cooperativo lo constituyen todas las cooperativas: mixtas y la matriz. Todas ellas están vinculadas a través de la 
participación de Eroski S. Cooperativa mediante la constitución de cooperativas mixtas por cada negocio.

EMES está guiado por principios y valores cooperativos. De ahí que todos los estatutos y reglamentos recogen dichos principios y valores como son la libre adhesión, organización democrática, participación en la gestión, soberanía del trabajo, retribución solidaria, carácter instrumental del capital, transformación social y educación. En cuanto a valores destacan la responsabilidad social, cooperación, participación, valor del consumidor e innovación.

Sin duda, no deja de ser un proyecto ambicioso la incorporación del personal contratado a la categoría de socios de trabajo. De resultar positiva esta incorporación, el grupo se beneficiaría con la aportación de capital de los nuevos socios, una mayor implicación de éstos con el proyecto empresarial, asunción de mayores grados de responsabilidad empresarial, mayor estabilidad en el empleo, etc. En definitiva, el resultado de todo este proceso redundará en el bien individual y colectivo del futuro de Eroski.

El proceso de cooperativización de Eroski se enfrenta a las duras consecuencias de la actual crisis económica. A pesar de la crisis, a comienzo del verano de 2012, 4.142 trabajadores por cuenta ajena englobados en GESPA habían accedido a la categoría de socio cooperativista. De todas formas, este proceso de cooperativización no ha hecho más que comenzar y el final, independiente del resultado, no dejaría de ser un intento serio de regenerar los valores y principios del cooperativismo.

\subsection{La experiencia autogestionaria de Irizar, S. Coop.}

\section{a) Datos de interés}

Irizar Sociedad Cooperativa fue creada en 1989 para fabricar carrocerías de autocares. Al inicio de su actividad fabricaba una amplia gama de carrocerías lo que causó unos deficientes resultados económicos.

Entre las cooperativas más participativas está Irizar, S. Coop. Esta cooperativa inicia el cambio de modelo de gestión con la incorporación del Sr. Saratxaga a la dirección de la empresa en 1991 cuando estaba en quiebra técnica y con un volumen de ventas de 20 millones REVESCO No 112 - Extraordinario en Homenaje al Profesor Alfonso Carlos MORALES GUTIÉRREZ - ISSN: 1885-8031 - www.ucm.es/info/revesco 
de euros y plantilla de 250 socios trabajadores. El cambio del modelo de gestión comenzó en 1993 después de fuertes debates. Desde entonces las cosa han cambiado sustancialmente y en la tabla de abajo se exponen algunos datos significativos de su evolución.

\begin{tabular}{lcccc}
$\begin{array}{l}\text { Año } \\
\text { (Millones de euros) }\end{array}$ & Ventas & Valor de los activos & Valor de recursos propios & Empleo \\
\hline 1991 & 20 & 10.000 & 8.000 & 250 \\
2000 & 250 & 255.000 & 140.000 & 1.700 \\
2006 & 430 & 464.000 & 285.000 & $3.030^{*}$ \\
\hline
\end{tabular}

Fuente: datos aportados por Irizar

*En la cooperativa matriz de Ormaiztegi (Gipuzkoa-País Vasco) 630 empleados.

El crecimiento del volumen de ventas ha sido constante, habiendo crecido el $20 \%$ en 2010 y el primer semstre de 2011 el 33\%, gracias al tirón de las ventas en el extranjero. Ha invertido en participación de en centros de investigación y en nuevos proyectos de autocares eléctricos. También sigue invirtiendo en países como Brasil, México, India, China, Marruecos, Sudáfrica, etc.

Con el nuevo modelo de gestión participativo y autogestionario comenzó el despegue de la cooperativa. Así, en 1995, se constituye una joint-venture en China al cincuenta por cien con el estado chino. Dos años más tarde, en 1997, se instala en Marruecos y amplía negocios en España, al año, siguiente en Brasil, creándose ese mismo año el Grupo Irizar. En 2001 comienza a construir carrocería en la India, al año siguiente adquiere otra empresa en España y, por último, en 2004 entra en Sudáfrica. Esta es la fotografía de la rápida expansión de la actividad de esta cooperativa donde se han roto los modelos de gestión tradicional, siendo sustituido por un modelo de gestión sistémico.

En el Estado español es el líder entre los fabricantes de carrocerías de autocares, habiendo construido 1.607 carrocerías y vendido 767 en 2005 , con una cuota de mercado del 45,7\%. Ese mismo año, en los centros de México, Brasil, Sudáfrica, Marruecos, India, China y en las factorías de Manresa y Sevilla fabricó 1.430 carrocerías. Los productos de esta cooperativa se encuentran en 71 países.

REVESCO No 112 - Extraordinario en Homenaje al Profesor Alfonso Carlos MORALES GUTIÉRREZ - ISSN: 1885-8031 - www.ucm.es/info/revesco 
Tanto para el caso de MCC en su conjunto como en el caso particular de Irizar, S. Coop. con un elevado grado de autogestión, las tasas de crecimiento de los negocios como de empleo son notables. De ello se puede extrapolar que la generalización de la innovación y la participación de los trabajadores en el conjunto de empresas del País Vasco, los niveles de crecimiento y generación de empleo podrían alcanza mayores cotas que las existentes en la actualidad

\section{b) Organización de la empresa}

El material recopilado de la cooperativa Irizar ( Agirre, 2006 y Bakaikoa, 2008)) constata que la persona es algo más que un recurso de trabajo, idea que defendía por el fundador de Mondragón Arizmendiarrieta desde los inicios de esta experiencia. Dejando actuar libremente a la persona, su capacidad creadora no tiene límites; pero hay que alejarse de las respuestas reproductoras simples. Asimismo, hay que confiar en las personas que participan en el proyecto y pensar que todo lo hace con la mejor buena voluntad; si una tarea la realiza mal, en lugar de reprenderla, hay que corregirla, puesto que una persona no se dedica voluntariamente a hacer las cosas mal. En suma, la confianza en las personas es básica para auto-organizarse en grupos, superando la idea clásica departamental-jerárquico, para dar entrada a equipos de trabajo compuestos por personas de ámbitos multidisciplinares autogestionados.

También es de destacar en Irizar que el poder no está jerarquizado, sino que se estructura en base a la idea de participación-liderazgo. En lugar del concepto jerárquico se parte del liderazgo. La formación de líderes debe ser la idea central de los órganos de la empresa. La idea del jefe con poderes ilimitados no existe; el jefe, en sentido tradicional, debe ser eliminado, y sustituido por el equivalente a entrenador de un equipo deportivo.

Se implanta la idea de trabajar en equipo donde se establecen relaciones horizontales. Los equipos de trabajo están constituidos por cuatro o cinco personas, aunque puede haber grupos hasta con quince miembros. Los componentes de estos grupos tienen nexos de algún tipo entre ellos y se buscará reforzar la cohesión grupal, así como entre los diferentes equipos porque se sabe que la colaboración es el secreto para el éxito. Asimismo, se implicará a los proveedores y clientes. 
En esta nueva organización, Irizar responde con tres tipos de grupos de trabajo estables. En primer lugar están los equipos línea-clientes constituidos por personas que participan en los trabajos del ciclo de la fabricación de cada autobús, sea su participación directa o indirecta, por lo que, en principio, se incluye todo el personal de la empresa cooperativa matriz de Irizar. En estos equipos participa el $90 \%$ de la plantilla y gestionan todo el proceso de fabricación de la carrocería, desde el pedido hasta la entrega al cliente del autocar.

Estos grupos de trabajo realizan a lo largo de toda la jornada las tareas planificadas. Con el fin de satisfacer los deseos de los clientes, están también en permanente contacto con los equipos de fiabilidad, puesto que éstos recogen las necesidades relacionadas con la calidad y servicio del producto fabricado por Irizar.

El líder de estos equipos anteriormente era elegido por sus miembros. Actualmente dicho líder es propuesto por el grupo coordinador y/o el grupo guía, y otras veces lo hacen los propios miembros del grupo línea-clientes.

En segundo lugar, los equipos de fiabilidad enlazan el mercado con los grupos líneaclientes. Las personas que forman parte de los grupos de fiabilidad están relacionadas con el mercado, puesto que cuanto más cerca se esté de los puntos de información de los mercados, más fácilmente se transmitirá al centro de producción. Es de subrayar que estos grupos son multidisciplinares y autogestionados, centrados en todo lo que rodea al mercado para que todos los flujos entre el mercado y centro de producción estén contemplados. En suma, conociendo las entrañas del mercado, será más fácil fidelizar al cliente y es para lo que se crean estos grupos cuyo ámbito de actuación se circunscribe a diferentes áreas geográficas (Francia-Holanda Bélgica; Polonia-República Checa-Eslovaquia-Hungría-Rusia; etc.).

Asimismo, estos equipos de fiabilidad lideran la estrategia de la empresa, definen objetivos tanto cualitativos como cuantitativos, conectan y traccionan los equipos de líneaclientes, comunican y comparten las necesidades del cliente a fin de lograr la estrategia mediante la visión de la empresa. 
Luego está el equipo guía de la cooperativa compuesto por los líderes de los 15 grupos de fiabilidad y los responsables del mercado de Europa del Norte y del Sur, reuniéndose una vez al mes para estudiar los avatares y la evolución del mercado.

También existe el grupo coordinador compuesto por los responsables de todos los grupos o elegidos por sus responsabilidades en el desarrollo de las diferentes tareas de la empresa. Su objetivo es hacer el seguimiento compartido de la producción y si se detectan disfunciones se trata de buscar soluciones lo más rápido posible; asimismo, este grupo impulsará la participación de todos los socios trabajadores de la cooperativa, informándoles veraz y puntualmente de todo lo acontecido alrededor de la misma, haciéndoles partícipes y parte integrante del proyecto global de Irizar.

El grupo guía no es estable como ocurre en las cooperativas de dirección clásica. Si se plantean nuevas iniciativas, el grupo guía se reformula con nuevos miembros.

En cuanto a participación de los miembros de los diferentes grupos, las encuestas dejan constancia de que la mayoría de los trabajadores participan en la definición de los objetivos de sus puestos de trabajo y de las tareas a realizar, así como en la fijación de los fines de la unidad de la empresa que forman parte. Otros trabajadores contestan diciendo que participan en la fijación de las tareas limitadas al ámbito de su puesto de trabajo donde aseguran que tienen autonomía suficiente para actuar.

A pesar del elevado grado de participación, las personas que trabajan en un grupo línea-cliente, tienen las tareas muy definidas (pintura, soldadura, etc.) por lo que no tienen demasiado campo para actuar. Sin embargo, en otros grupos más genéricos (grupos de gestión), las posibilidades de actuar, hacer propuestas de mejora, etc. son más reales. Una vez desarrollado el proyecto por parte de los grupos creados "ad hoc", éste se disuelve. En estos grupos, los miembros participantes desarrollan sus capacidades y realizan contribuciones a la empresa, superando en muchas ocasiones las previsiones iniciales.

De esta forma se quiere romper con la monotonía del trabajo y en lugar de hacer siempre las mismas tareas se posibilita que los trabajadores desarrollen iniciativas generadoras de valor añadido. 
¿Por qué se funciona así? La respuesta de los líderes de Irizar, S. Coop. entrevistados es rotunda: extraer el mayor rendimiento a la energía atomizada existente en la cooperativa, convirtiendo en viable cualquier proyecto y ello, a largo plazo, redunda en la mejora de la competitividad.

Otro aspecto es el de la información de la empresa. En la empresa de gestión tradicional la información es unidireccional. En un proyecto compartido como en la cooperativa Irizar, se comparten todos los datos económicos, se plantean las ideas de futuro de la empresa sobre la dirección que debe tomar para seguir siendo competitivos, etc. Por tanto, una buena comunicación es un factor primordial para el buen funcionamiento de la empresa autogestionada.

Para tener éxito la comunicación se establecen foros donde participan todos los que quieran acudir a los mismos; incluso pueden ser convocados por iniciativa particular. Según la encuesta, el $42 \%$ dice haber participado en múltiples iniciativas, habiendo coordinado reuniones sobre materias relacionadas con las responsabilidades que tienen en la producción de la empresa.

También funcionan redes de comunicación entre distintos grupos de línea-clientes, de modo que intercambian información de lo que cada grupo realiza, sus problemas, soluciones planteadas, etc. En suma, la comunicación en red es fluida y permanente, tanto de la situación económica de la empresa como de los problemas planteados en la fabricación.

Además de los mencionados foros puntuales, se convocan foros de discusión de temas básicos concernientes a la empresa tres veces al año. Estas reuniones son equivalentes a la Asamblea General. En las dos primeras reuniones generales se debaten y reflexionan temas relacionados con el ejercicio económico, presentándose en abril los resultados como en cualquier cooperativa. En la Asamblea General ordinaria participan los miembros legales, los socios, mientras que en las restantes reuniones pueden acudir todos los trabajadores, permanentes y temporales, con voz y voto.

Así mismo, todos los días al inicio de la jornada laboral se celebra la reunión del día. Esta reunión la coordina un miembro del grupo de fiabilidad, participando en la misma todos los responsables de las diferentes secciones de cada grupo de la línea-cliente. En esta reunión 
se analiza la situación del autobús que está en proceso de fabricación, sus problemas para darle una solución rápida y poner el autocar a disposición del cliente.

Los problemas planteados en dicha reunión se analizan en otra reunión la cual se celebra más tarde donde participan los seis coordinadores de las reuniones de cada línea de producción, además del coordinador del personal, el coordinador de la producción, los responsables del mercado, así como los miembros de los servicios tecnológicos.

Otro foro es el de la planificación el cual se celebra los viernes y tiene la finalidad de planificar la producción de la semana siguiente, así como hacer las previsiones para 2-3 semanas posteriores. Además de estos foros presenciales, también funciona el sistema intranet y la revista trimestral, todo lo cual redunda en el perfeccionamiento de la información de la empresa. Por último, es de destacar que cualquier miembro puede impulsar la creación de foros presenciales o virtuales para tratar cuestiones relacionadas con el funcionamiento de la empresa.

Es de subrayar, asimismo, la libertad y autonomía de los miembros/equipos de Irizar, puesto que son ellos los administradores de su tiempo de trabajo. El inicio de la actividad de cada grupo lo fija el grupo, pero teniendo en cuenta que el trabajo se desarrolla en cadena; en principio no hay hora para iniciar ni finalizar la jornada, sólo se deben cumplir los objetivos establecidos. No existe el control de asistencia, sólo se limita por la responsabilidad de los miembros tal como afirma el $64 \%$ de los encuestados. En otras empresas no se les reconoce esta libertad a los trabajadores.

Antes a los trabajadores se les daba un plan por escrito de lo que tenían que hacer cada uno. Ahora el trabajo lo distribuyen en el grupo. Cada persona, dependiendo de su habilidad, realiza sus tareas a ritmos diferentes y si necesita ayuda, ésta le será proporcionada por el resto de miembros del grupo.

Las normas de trabajo se dejan de lado y en su lugar se potencia el debate. El procedimiento burocrático se debe evitar y se toma como base para avanzar el conflicto (Ugarte, 2004: 327), conflicto causado por las discusiones y deber ser. Hay diversidad de personas, así como desacuerdos lo que hace que se deben respetar ambos aspectos. Y el directivo debe demostrar que está para ayudar y al servicio de otros. 
Los socios trabajadores son dueños y así se sienten en el ámbito de la gestión y en la acción. El tener autonomía y libertad de acción les hace sentirse dueños del proceso de fabricación del autobús y ello les identifica con la empresa. No sólo son propietarios del capital, sino también del proceso productivo; este sentimiento de pertenencia facilita el estar tomando decisiones permanentemente.

El sistema de gestión tradicional significa cumplir a rajatabla las decisiones impuestas desde la jerarquía; ahora necesitan información que algunas veces es demasiado abundante y caótica, pero con el tiempo se selecciona toda la información recibida de otros grupos, así como de los clientes y proveedores. Es opinión de socios trabajadores que se informa de todo, no hay cosas escondidas. A veces es información redundante, pero siempre positivo.

En Irizar no hay un poder impuesto, por ello se celebran tantas reuniones e intercambio de información. En lugar de mando hay liderazgo, desarrollándose el poder de forma horizontal y descentralizada. Las decisiones son tomadas entre muchas personas; de no ser así, la autogestión/autoorganización no sería posible. Ello significa: trabajar en grupo con capacidad y disposición de comunicar, y tomar decisiones y asumir riesgos.

Por otra parte, Irizar no realiza Planes de Gestión, sino que parte de unas ideas básicas, simples, lo que constituye el denominado Pensamiento Estratégico. Son pocas las ideas compartidas, pero abiertas para desarrollarlas; son ideas compartidas y debatidas, pero antes de eso el grupo coordinador de Irizar las ha discutido y reflexionado durante mucho tiempo.

Las inversiones para el ejercicio siguiente se realizan en función de lo que dicen los clientes, etc. y lo propuesto se relacionará con las inversiones mencionadas. Por otra parte, estas inversiones se acuerdan en el grupo coordinador y el grupo guía y luego se presentan en la correspondiente Asamblea General para su aprobación. También se debe recabar información del mercado por medio de los grupos de fidelidad y de los distribuidores para compartir las ideas de todos.

Todos los años se revisan las ideas centrales y es el grupo que coordina la dirección el que inicia dicha revisión, generalizando más tarde para el resto del personal. Hay que comunicar estas ideas básicas a todos los miembros de Irizar para su posterior estudio. 
En Irizar es importante la educación y la preparación. La primera es adquirida con la confianza y cercanía con el resto de personas y la segunda consiste en obtener el mayor conocimiento. La educación cambia la forma de ser y de hacer de las personas. El actuar en grupo es educativo.

El pertenecer al grupo línea-cliente hace que sus miembros tengan conocimiento de lo que están haciendo en la producción y eso le da sentido a su trabajo. Ello les hace estar unidos y orgullosos de trabajar en este proyecto autogestionado, aunque el sistema en sí es inestable, pero hace avanzar.

\subsection{La experiencia de disidentes: GBE-NER}

\section{a) Datos de interés}

GBE-NER, acrónimo, en euskera y castellano, de Nuevo Estilo de Relaciones, es una asociación sin ánimo de lucro que agrupa en la actualidad veinte organizaciones de muy diverso tipo, tanto en lo que se refiere a los sectores de actividad, como en cuanto a su forma jurídica o incluso respecto al lucro ${ }^{4}$. Desde noviembre de 2010 estas organizaciones que comparten el Nuevo Estilo de Relaciones se han agrupado en este grupo para poner en marcha proyectos con el fin de lograr mejoras para todas las empresas asociadas.

El año 2008 el conjunto de estas empresas facturaba 550 millones de euros y dos años más tarde esa cifra, como consecuencia de la crisis económica, se situaba en 360 millones de euros, un 35\% menor. El índice de actividad en 2010 era del 65\% y la plantilla, que en 2008 era de 2.420 personas, descendió sólo en 100 puestos de trabajo, algo más del 4\%; en 2011 ha subido hasta 2.400 empleos.

Se espera que en 2011 las ventas aumenten en un 25\% respecto al año anterior y los excedentes alcancen los 26 millones de euros, después de haber obtenido 10 millones el año anterior, teniendo presencia en 60 países.

La visión que comparten, tal como se define en su página web, es:

\footnotetext{
${ }^{4}$ Entre las organizaciones predominan las empresas pero también hay una organización sin ánimo de lucro, Urtxintxa.
} 
Ser una asociación de personas que sitúan el conocimiento por delante del capital, que conviven con la naturaleza y que creen en la eficiencia y la generosidad que posibilitan el desarrollo de una sociedad integradora y equilibrada.

A fecha de hoy, "la historia de GBE-NER está todavía por escribir". Las organizaciones que se han agrupado comparten "de forma libre un nuevo estilo de relaciones". Las ideas básicas de este innovador proyecto empresarial están recogidas en el libro de Koldo Saratxaga ${ }^{5}$, anterior líder de la Sociedad Cooperativa Irizar que pertenecía a la Coporación Mondragón. La estructura es mínima y las actividades que acuerdan emprender se llevan a cabo con los recursos de las diversas organizaciones pertenecientes al grupo.

La asociación se mantiene a través de las contribuciones de sus organizaciones socias en tanto que cada una de ellas debe destinar el $5 \%$ de sus ingresos y el $2 \%$ de su tiempo a GBE-NER. Además, en GBE-NER group los distintos proyectos que se van poniendo en marcha tienen autonomía y pueden abarcar áreas muy diversas, desde la búsqueda específica de ahorros en costes hasta la identificación de oportunidades de mercado o la generación de nuevos negocios y, sobre todo, compartir experiencias.

\section{b) Definición del proyecto}

GBE-NER nace como organización vinculada a una misión, una visión, unos valores y unos objetivos organizativos concretos, que pueden consultarse en su página web. En esencia pretende desarrollar un "nuevo estilo de relaciones". Destaca sobremanera el papel central que asignan a la persona, no como un recurso, sino de una forma muy parecida a la que se maneja en el entorno familiar. Se insiste en la importancia de "no despedir" a ninguna persona. Esta innovadora organización requiere personas que se comprometan de forma permanente con la misma. Asimismo, se parte de que a la persona le es inherente trabajar en equipo. Así, cuando exponen los valores de gbe-ner, se dice:

La estructura jerárquica debe dar paso al trabajo en equipo. Todas las personas somos portadores de valores y capacidades, sin embargo, el modelo

\footnotetext{
${ }^{5}$ SARATXAGA, K. (2007): Un nuevo estilo de relaciones para el cambio organizacional pendiente, Prentice Hall, Madrid.
} 
imperante las clasifica y decide quién piensa y ordena, y quién es controlado y realiza, lo cual nos lleva a la repetición de tareas, a la falta de motivación, a la falta de creatividad.

Lo más preciado que tenemos en las organizaciones son las personas. La solución es implicar a las personas y que se junten, que definan sus objetivos, sus retos, que liberen su tiempo de tareas anodinas, que adquieran nuevos conocimientos y que sueñen y creen su propia realidad y su propia verdad.

Los objetivos del grupo consisten en gestionar y desarrollar proyectos conjuntos, gestionar compras y suministros en común, de forma que pueden obtener mejores precios, permitir reubicaciones de los trabajadores, siguiendo la política de los excedentes laborales de la corporación Mondragón, y negociar conjuntamente servicios financieros en mejores condiciones. En suma, el grupo se beneficiará por su mayor capacidad de negociación y ello le acompañará el valor solidario cuando surjan excedentes laborales debido a la crisis, innovación o constreñimiento de mercados.

\section{c) Modelo de gestión}

GBE-NER es una iniciativa que, como tal, asienta sus principios de gestión no en un "modelo" sino en un "estilo". El lenguaje es importante. No se habla de "empresa" sino de "proyecto". Las personas, situadas en el centro de este estilo, comparten los beneficios que se obtienen en cada proyecto.

De la asociación surgen equipos de trabajo, que es la forma natural en que se pretende potenciar a la persona. Son equipos que buscan sinergias y oportunidades de mejora para las organizaciones asociadas. Cada una de ellas desarrolla el estilo de relaciones que define a GBE-NER. En él es muy importante la comunicación, intensa y sincera, entre todas las personas. Esto se traduce, por ejemplo, en el uso intensivo de las asambleas. El compromiso debe ser expreso para cumplir con la misión que se asignan, "de personas para personas que crecen abiertas al mundo", como subrayan.

El hilo conductor de las organizaciones que conforman GBE-NER es el apoyo en equipos autogestionados, donde el control debe dejar paso a la comunicación, la libertad y la 
responsabilidad. Cada organización busca la conexión con sus clientes y proveedores más cercanos, tratando de integrarlos en su estilo de relaciones ${ }^{6}$. De ahí que sea habitual que, por ejemplo, los clientes lleguen a participar en ciertos equipos de planificación, tal como implantó Saratxaga en la Sociedad Cooperativa Irizar.

\section{d) Organización y cultura corporativa}

Hablar de GBE-NER es hablar del estilo de relaciones que define a las entidades que conforman la asociación. Cada una de ellas busca el compromiso de sus personas con un proyecto compartido, que supone una labor de siembra con la mirada puesta en un futuro que está por construir y del que las personas deben sentirse propietarias y co-responsables.

En tanto que la gran mayoría de las entidades que la componen son organizaciones con ánimo de lucro, se realiza una labor muy intensa para que todas las personas comprendan qué quiere decir esto y cómo se obtienen los beneficios suficientes para recompensar adecuadamente el esfuerzo que se desarrolla. Algo que define este estilo es el reparto de los beneficios económicos entre todas las personas que comparten el proyecto. En cierto modo hay una clara aceptación del hecho económico asociado de forma indivisible a la idea de eficiencia como condición para la supervivencia de la empresa. Intentan crear la máxima transparencia en lo económico y explicar a las personas que "no pueden cumplir el objetivo social si no consiguen los resultados económicos". También es de reseñar el esfuerzo que se hace en trabajar con indicadores tanto tangibles como intangibles y datos que las personas deben comprender para saber qué hacer para impactar en ellos.

El estilo de relaciones dentro de cada una de las organizaciones pasa también por una intensa labor de despliegue de distintos tipos de equipos autogestionados dentro de cada organización. Esos equipos trabajan con un Plan de Ideas y Objetivos (PIO) y se organizan de manera autónoma para llevar a cabo las actividades que en otras organizaciones suelen traducirse en áreas funcionales y departamentos.

Así pues, las personas -insistiendo en que somos diferentes pero que debemos compartir el proyecto-, los equipos autogestionados y el uso de las asambleas para evitar

\footnotetext{
${ }^{6}$ El estilo de GBE-NER rehuye el uso de anglicismos (como sería el caso de stakeholder por ejemplo). Se esfuerzan por la introducción de un lenguaje más directo y llano que facilite la comunicación entre las partes.
} 
intermediaciones, definen el estilo de gestión de las organizaciones de GBE-NER. Respecto a las personas, se insiste en que todas nos distribuimos de acuerdo con una campana de Gauss y que no se trata tanto de apartar a quienes están "en la cola", sino de desplazar en conjunto la campana hacia el lado positivo.

\section{e) Mirando al futuro}

Como decíamos al principio, GBE-NER tiene toda una historia por escribir. Es de reciente constitución (2010) y el tiempo irá evidenciando sus logros y aportaciones. Desde luego que tienen vocación de futuro a fin de desarrollar proyectos para "construir una nueva sociedad" que esté basada en este "nuevo estilo de relaciones" que da nombre y fundamenta a la asociación. Ésta ha publicado varios libros donde se recogen las actividades llevadas a cabo los planteamientos de la nueva organización de empresas.

Las áreas de actuación de GBE-NER son amplias y diversas: los derechos humanos, el medio ambiente, la gobernanza, el desarrollo humano sostenible, las personas y la sociedad en su conjunto. Como dicen en su web, "será el proceso social, humano, político en su sentido amplio, cultural, económico, ambiental, lo que determine, en su momento, las actuaciones concretas que llevaremos a cabo".

El proyecto empresarial se fundamenta sobre el principio de solidaridad y autogestión lo que dará carácter a GBE-NER cuando todos los grupos empresariales, aprovechan las llamadas oportunidades surgidas por la crisis económica, para abaratar los costes laborales, cuando no la eliminación de los puestos de trabajo, este grupo trata de poner en práctica el modelo de la corporación Mondragón, consistente en reubicar los excedentes laborales. No se expulsa a ningún trabajador porque eso significaría una descapitalización de la empresa y, en última instancia iría en detrimento de la sociedad ya que el desempleado drenaría fondos públicos.

\section{CONCLUSIONES}

La evidencia nos dice que las empresas cooperativas a medida que se desarrollan y los fundadores se van apartando de la actividad empresarial, muestran signos de deterioro de los principios que pusieron en marcha el proyecto cooperativo. Hay abundantes autores que han 
analizado este fenómeno de alejamiento desde los principios lo cual hace que las nuevas generaciones de socios, sean de consumo de crédito o trabajadores, ni se plantean y algunas veces ni saben que son socios de la empresa cooperativa. En casos de socios trabajadores de cooperativas de trabajo asociado, más bien funcionan como funcionarios poseedores de su puesto de trabajo y que en algunas experiencias adquieren dicha condición por ser descendientes de socios que abandonan la empresa por jubilación.

Contra esta degradación de los valores y principios cooperativos, en el País Vasco ha surgido una corriente que trata de corregir esta deformación cooperativa. El liderazgo de dicha corriente lo conduce El Consejo Superior de Cooperativas de Euskadi. Este Consejo, órgano máximo de promoción y difusión de valores y principios cooperativos, trata de profundizar los mismos, para luego implementarlos en las cooperativas de ámbito vasco, sobre todo, a través de la Confederación de Cooperativas de Euskadi, asociación que agrupa a la mayoría de cooperativas del País Vasco.

Independientemente de la iniciativa del Consejo, existen varios casos que tratan de impulsar la aplicación de los valores y principios cooperativos. De los tres casos expuestos en este análisis, destaca el de la Sociedad Cooperativa Irizar donde se está impulsando, con éxito, un sistema autogestionario donde participan los socios trabajadores y clientes aportando sus ideas y soluciones, con el fin de mejorar el producto final. En la base del proyecto empresarial está la persona y su desarrollo como ser humano mediante el trabajo. Los datos económicos avalan el éxito empresarial de Irizar.

El segundo caso analizado es el del grupo de distribución EROSKI donde la empres matriz es una cooperativa de consumidores y de trabajo asociado. El crecimiento de este grupo comenzó a principios de los noventa y no ha parado hasta 2010 debido a la crisis económica y alguna inversión estratégica. El crecimiento se ha realizado a partir de la cooperativa matriz, por irradiación, pero la gran mayoría de empresas creadas han sido sociedades de capital. Desde 2007 se trata de que estas sociedades de capital se transformen en cooperativas donde la cooperativa matriz tenga el 50\% del capital y el resto lo aporten los socios trabajadores. En suma, que todos los trabajadores por cuenta ajena se trasformen en socios trabajadores, lo que significaría un paso hacia la asunción de principios cooperativos, haciéndoles partícipes de la propiedad y resultados. En suma, el grupo se cooperativizaría. 
El último caso estudiado es un conglomerado de empresas donde forman parte sociedades de capital (anónimas y limitadas), sociedades laborales y cooperativas de trabajo asociado. Sin embargo, a pesar de esta diversidad de figuras jurídicas, el grupo funciona con principios de solidaridad e intercooperación. De esta forma, GBE-NER está siendo capaz de absorber los excedentes laborales del grupo así como para fomentar un fondo de solidaridad para hacer frente a los riegos causados por las crisis económicas.

\section{BIBLIOGRAFÍA}

AGIRRE, A., (2006), Kooperatiben koeboluzioa konplexutasunaren paradigmatik: beren idiosinkrasiari eusteko bidea, Tesis doctoral, Mondragón Unibertsitatea.

ALTUNA, L. (coord.) (2008): La Experiencia Cooperativa de Mondragón. Una síntesis general. Eskoriatza. LANKI, Universidad de Mondragon.

AZKARRAGA, J. (2007): Mondragon ante la globalización. La cultura cooperativa ante el cambio de época. Eskoriatza. LANKI, Universidad de Mondragon.

BAKAIKOA, et al, (2008), "The Neo-Schumpeterian model of economic development in the Basque Country: the role of Social Economy", Ciriec-España, Revista de Economía Pública, Social y Cooperativa, ${ }^{\circ} 62$.

BAKAIKOA, B. et al, (2004), "Governance of the Mondragon Corporación Cooperativa", Annals of Public and Cooperative Economics, vol.75, $\mathrm{n}^{\circ} 4$.

BAKAIKOA, B. (1995), El cooperativismo vasco y el año 2000, Marcial Pons, Madrid.

BORZAGA, C. y DEPEDRI, S. (2012): "The emergence, institutionalisation and challenges of social enterprises: the Italian experience", CIRIEC-España, Revista de Economía Pública, Social y Cooperativa, No 75, pp. 35-53.

BORZAGA, C. y SANTUARI, A., (2001), "Italy: from traditional cooperatives to innovative social enterprises", The emergence of Social Enterprises, Routledge, London

CHAVES, R. et al, (2004), "Governance and Management in the Social Economy", Annals of Public and Cooperative Economics, vol.75, $\mathrm{n}^{\circ} 1$.

Consejo Superior de Cooperativas de Euskadi, (2009), Reflexión Estratégica, CSCEEKGK,Vitoria-Gasteiz.

Consejo Superior de Cooperativas de Euskadi, (2010), Plan de Gestión, 2010 y 2011, CSCEEKGK. Vitoria-Gasteiz.

CORNFORTH, C. et al, (1988), Developing Successful Worker Cooperatives, Sage, London. REVESCO No 112 - Extraordinario en Homenaje al Profesor Alfonso Carlos MORALES GUTIÉRREZ - ISSN: 1885-8031 - www.ucm.es/info/revesco 
Diario Cinco Días, 19 y 20 de marzo, 2011.

DIMAGGIO, P. y POWEL, W. (1983): “The Iron Cage Revisited: Institutional Isomorphism and Colletive Rationality in Organizational Fields", American Sociological Review, №48, pp. 147-160.

DEFOURNY, J. y THOMAS, H. (1986): "Report of the Fourth International Conference on the economics of self-management" Annals of Public and Cooperative Economics, Volume 57, Issue 1, pp. 117-126.

EVERS, A. y LAVILLE, J.L. (2004): "Defining the Third Sector in Europe", en Evers, A. y Laville, J.L. (ed.): The Third Sector in Europe, Cheltenham: Edward Elgar publishing, pp. $11-42$.

ERRASTI et al, (2003), "The internationalization of cooperatives: the case of Mondragon Cooperative Corporation", Annals of Public and Cooperative Economics, vol.74, nº 4.

GALBRAITH, J., (1967), The new industrial state, Houghton Mufflin, Boston.

MEISTER, A., (1974), Le participation dans les associations, Editions Ouvriéres, Paris.

MEISTER, A., (1984), Participation, Associations, Development and Change, Transaction, New Brunswick, NJ.

MICHELS, R., (1949), Political Parties: a Sociological Study of Oligarchical Tendencies of Modern Democracy, Free Press, New York.

PESTOFF, V. (2009), "Social Enterprise, the Third Sector, Democracy and the Welfare State: a Scandinavian Perspective", $2^{\text {nd }}$ Ciriec-International Research Conference of Social Economy (CD), Östersund (Suecia), ISBN: 978-91-978685-0-1.

SARATXAGA, K. (2007): Un nuevo estilo de relaciones para el cambio organizacional pendiente, Prentice Hall, Madrid

SINGER, P., (2000), Economía Solidaria: posibilidades y desafíos, Conferencia al Sindicato de Ingenieros, Ediciones Contexto, Ría Janeiro

SPEAR, R; CORNFORTH, C y AIKEN, M.: (2009): "The governance challenges of social enterprises: evidence from a UK empirical study", Annals of Public and Cooperative Economics, Volume 80, Issue 2, pp 247-273.

SPEAR, R., (2004), "Governance in Democratic Member-Based Organisations", Annals of Public and Cooperative Economics, vol.75, $\mathrm{n}^{\circ} 4$.

TULANKIDE Revista de Actualidad de la Corporación Mondragón (www.tulankide.com)

REVESCO No 112 - Extraordinario en Homenaje al Profesor Alfonso Carlos MORALES GUTIÉRREZ - ISSN: 1885-8031 - www.ucm.es/info/revesco 
UGARTE, L., (2004), ¿Sinfonía o Jazz? Koldo Saratxaga y el Modelo Irizar, Granica, Barcelona.

REVESCO No 112 - Extraordinario en Homenaje al Profesor Alfonso Carlos MORALES GUTIÉRREZ - ISSN: 1885-8031 - www.ucm.es/info/revesco 